\title{
Positive linear maps on normal matrices
}

\author{
Jean-Christophe Bourin* and Eun-Young Lee ${ }^{\dagger}$
}

\begin{abstract}
For a positive linear map $\Phi$ and a normal matrix $N$, we show that $|\Phi(N)|$ is bounded by some simple linear combinations in the unitary orbit of $\Phi(|N|)$. Several elegant sharp inequalities are derived, for instance for the Schur product of two normal matrices $A, B \in \mathbb{M}_{n}$,

$$
|A \circ B| \leq|A| \circ|B|+\frac{1}{4} V(|A| \circ|B|) V^{*}
$$

for some unitary $V \in \mathbb{M}_{n}$, where the constant $1 / 4$ is optimal.

Keywords. Matrix inequalities, Positive linear maps, Schur products, unitary orbits, matrix geometric mean.

2010 mathematics subject classification. 47A30, 15A60.
\end{abstract}

\section{Introduction}

Let $\mathbb{M}_{n}$ denote the space of complex $n \times n$ matrices and let $\mathbb{M}_{n}^{+}$stands for the positive (semi-definite) cone. A linear map $\Phi: \mathbb{M}_{n} \rightarrow \mathbb{M}_{m}$ is called positive if $\Phi\left(\mathbb{M}_{n}^{+}\right) \subset \mathbb{M}_{m}^{+}$. A nice exposition of these maps on matrix algebras can be found in Bhatia's book [1]. The Schur product with $A \in \mathbb{M}_{n}^{+}, X \mapsto A \circ X$ is a basic example of positive linear map on $\mathbb{M}_{n}$ (Schur's theorem; for which it suffices to check that the Schur product of two rank one positive matrices is again a rank one positive matrix).

A famous Schwarz inequality due to Man-Duen Choi [4, and previously to Kadison for the Hermitian case, says that

$$
\Phi\left(N^{*}\right) \Phi(N) \leq \Phi\left(N^{*} N\right)
$$

for all normal matrices $N$ in a unital matrix algebras $\mathcal{A}$ on which acts the unital positive linear mas $\Phi$. Written as $|\Phi(N)|^{2} \leq \Phi\left(|N|^{2}\right)$, this is a noncommutative Jensen inequality for the convex function $z \mapsto|z|^{2}$ on $\mathbb{C}$. Choi's inequality is related to another classical fact, the Russo-Dye theorem [6] stating that unital positive linear maps are contractive, or more generally, that any positive linear maps attains its norm at the identity.

Comparing $|\Phi(N)|$ and $\Phi(|N|)$ via operator inequalities seems more delicate. In this note, we aim to obtain such comparisons by using additive combination in the unitary

*This research was supported by the French Investissements dAvenir program, project ISITE-BFC (contract ANR-15-IDEX-03).

$\dagger$ This research was supported by Basic Science Research Program through the National Research Foundation of Korea (NRF) funded by the Ministry of Education (NRF-2018R1D1A3B07043682) 
orbits of $\Phi(|N|)$. As a consequence we point out some refinements of the classical RussoDye theorem stating that any positive linear maps attains its norm at the identity. The results may also be useful to obtain some estimates for various non $\mathbb{C}$-linear maps; we close this introduction by providing such an example. For $A=\left(a_{i, j}\right) \in \mathbb{M}_{n}$, we consider the conjugate matrix $\bar{A}:=A^{* T}=\left(\overline{a_{i, j}}\right)$, and the matrix with the real parts, $A_{\mathbb{R}}:=(A+\bar{A} / 2$.

Proposition 1.1. If $A$ is a normal matrix, then $\left|\operatorname{det} A_{\mathbb{R}}\right| \leq \operatorname{det}|A|_{\mathbb{R}}$.

This is a consequence of Corollary 2.4. This inequality does not hold for some nonnormal two-by-two matrices.

\section{Positive linear maps}

We first single out the simplest case of our main theorem.

Proposition 2.1. Let $\Phi: \mathbb{M}_{n} \rightarrow \mathbb{M}_{m}$ be a positive linear map and let $N \in \mathbb{M}_{n}$ be normal. Then, there exists a unitary $V \in \mathbb{M}_{m}$ such that

$$
|\Phi(N)| \leq \frac{\Phi(|N|)+V \Phi(|N|) V^{*}}{2}
$$

Thus, $|\Phi(N)|$ is dominated by the mean of two elements in the unitary orbit of $\Phi(|N|)$. There exists a whole family of such sharp inequalities, this is the content of the following main theorem. $R \in \mathbb{M}_{n}$ is called a reflexion if $R^{2}=I$.

Theorem 2.2. Let $\Phi: \mathbb{M}_{n} \rightarrow \mathbb{M}_{m}$ be a positive linear map, $n, m \neq 1$, and let $N \in \mathbb{M}_{n}$ be normal. Fix $\beta>0$. Then, there exists a unitary $V \in \mathbb{M}_{m}$ such that

$$
|\Phi(N)| \leq \beta \Phi(|N|)+\frac{1}{4 \beta} V \Phi(|N|) V^{*}
$$

If $\beta \geq 1 / 2$, then the constant $1 / 4 \beta$ is the smallest possible one and this inequality is sharp even if we confine $N$ to the class of Hermitian reflexions in $\mathbb{M}_{n}$.

$A$ refinement of this inequality is the double inequality

$$
|\Phi(N)| \leq \Phi(|N|) \# V \Phi(|N|) V^{*} \leq \beta \Phi(|N|)+\frac{1}{4 \beta} V \Phi(|N|) V^{*}
$$

where \# stands for the usual geometric mean.

We do not know wether the theorem is sharp or not when $0<\beta<1 / 2$. A reader familiar with the theory of positive maps will note that, in the course of the proof, we quickly establish a basic fact due to Stinespring [7] stating that positive maps defined on a commutative domain are completely positive. 
Proof. Consider the spectral decomposition of $N$,

$$
N=\sum_{i=1}^{n} \sigma_{i} E_{i}
$$

where $\sigma_{i}$ runs over the eigenvalues of $N$ counted with their multiplicities and $E_{i}$ are rank one projections, $i=1, \ldots, n$. Note that $E_{i}=x_{i} x_{i}^{*}$ for some column vectors $x_{i} \in \mathbb{M}_{n, 1}$. Let $\mathcal{N}$ be the $*$-commutative subalgebra spanned by the projections $E_{i}, i=1, \ldots, n$. When restricted to $\mathcal{N}$, the map $\Phi$ has the form

$$
\Phi(X)=\sum_{i=1}^{m} \sum_{j=1}^{n} Z_{i, j}^{*} X Z_{i, j}
$$

for some rank 1 or 0 matrices $Z_{i, j} \in \mathbb{M}_{n, m}, i=1, \ldots, n, j=1, \ldots, m$. Indeed, the map $\Phi$ on $\mathcal{N}$ is determined by its values on the projections $E_{i}$, hence, setting $Z_{i, j}=x_{i} R_{i, j}$ where $R_{i, j} \in \mathbb{M}_{1, m}$ is the $j$-th row of $\Phi\left(E_{i}\right)^{1 / 2}$, we obtain the representation (2.1).

This decomposition of $\Phi$ on $\mathcal{N}$ as a sum of congruences maps ensures that

$$
\left(\begin{array}{cc}
\Phi(A) & \Phi(B) \\
\Phi\left(B^{*}\right) & \Phi(C)
\end{array}\right) \geq 0
$$

whenever the matrices $A, B, C$ in $\mathcal{N}$ satisfy

$$
\left(\begin{array}{ll}
A & B \\
B^{*} & C
\end{array}\right) \geq 0
$$

In particular, this happens if $A=C=|N|$ and $B=N$, as

$$
\left(\begin{array}{cc}
|z| & z \\
z^{*} & |z|
\end{array}\right) \geq 0
$$

for all $z \in \mathbb{C}$. Therefore

$$
\left(\begin{array}{cc}
\Phi(|N|) & \Phi(N) \\
\Phi\left(N^{*}\right) & \Phi(|N|)
\end{array}\right) \geq 0
$$

Now, let $V^{*}$ be the unitary part in the polar decomposition $\Phi(N)=V^{*}|\Phi(N)|$. We have, for all $\alpha>0$,

$$
\left(\begin{array}{ll}
\alpha^{-1 / 2} V & -\alpha^{1 / 2} I
\end{array}\right)\left(\begin{array}{cc}
\Phi(|N|) & \Phi(N) \\
\Phi\left(N^{*}\right) & \Phi(|N|)
\end{array}\right)\left(\begin{array}{c}
\alpha^{-1 / 2} V^{*} \\
-\alpha^{1 / 2} I
\end{array}\right) \geq 0 .
$$

Equivalently,

$$
|\Phi(N)| \leq \frac{\alpha \Phi(|N|)+\alpha^{-1} V \Phi(|N|) V^{*}}{2}
$$

and setting $\beta=\alpha / 2$ yields the inequalities of the lemma.

A map $\Phi: \mathbb{M}_{n} \rightarrow \mathbb{M}_{m}$ induces, by taking compressions to the upper left $2 \times 2$ corners, a map from $\mathbb{M}_{2}$ to $\mathbb{M}_{2}$. Therefore, to check that the constant $1 / 4 \beta$ is optimal when $\beta \geq 1 / 2$, we may assume $n=m=2$. Thus suppose $\beta \geq 1 / 2$, consider

$$
A=\left(\begin{array}{cc}
2 \beta & 1 \\
1 & (2 \beta)^{-1}
\end{array}\right)
$$


and the positive linear map on $\mathbb{M}_{2}, Z \mapsto A \circ Z$, and take the reflexion

$$
R=\left(\begin{array}{ll}
0 & 1 \\
1 & 0
\end{array}\right) .
$$

We claim that the inequality, for any unitary $V \in \mathbb{M}_{2}$ and any $0<c<1$,

$$
|A \circ R|-\beta(A \circ|R|) \leq \frac{c}{4 \beta} V(A \circ|R|) V^{*}
$$

cannot hold, so that the constant $1 / 4 \beta$ is optimal in our theorem. Indeed, using $\beta \geq 1 / 2$, we have

$$
\lambda_{1}(|A \circ R|-\beta(A \circ|R|))=\frac{1}{2}, \quad \lambda_{2}(|A \circ R|-\beta(A \circ|R|))=1-2 \beta^{2},
$$

and

$$
\frac{c}{4 \beta} \lambda_{1}(A \circ|R|)=\frac{c}{2}, \quad \frac{c}{4 \beta} \lambda_{2}(A \circ|R|)=\frac{c}{8 \beta^{2}} .
$$

As (2.3) entails

$$
\frac{1}{2}=\lambda_{1}(|A \circ R|-\beta(A \circ|R|)) \leq \frac{c}{4 \beta} \lambda_{1}(A \circ|R|)=\frac{c}{2}
$$

we necessarily have $c \geq 1$ in (2.3).

To get the double inequality involving the geometric mean \#, consider again the block-matrix (2.2) and apply a unitary congruence with

$$
\left(\begin{array}{ll}
V & 0 \\
0 & I
\end{array}\right)
$$

where $V^{*}$ is still the unitary factor in the polar decomposition of $\Phi(N)$. This shows that

$$
\left(\begin{array}{cc}
V \Phi(|N|) V^{*} & |\Phi(N)| \\
\left|\Phi\left(N^{*}\right)\right| & \Phi(|N|)
\end{array}\right) \geq 0
$$

It then follows from the maximal property of \# that

$$
|\Phi(N)| \leq V \Phi(|N|) V^{*} \# \Phi(|N|)
$$

which is exactly the first inequality in the double inequality. The second one is a simple application of the arithmetic-geometric means inequality.

Several eigenvalue inequalities follow from the theorem combined with some basic relations of Weyl type.

Corollary 2.3. Let $\Phi: \mathbb{M}_{n} \rightarrow \mathbb{M}_{m}$ be a positive linear map, $n, m \neq 1$, and let $N \in \mathbb{M}_{n}$ be normal. Then, for all $\beta>0$ and all integers $j, k \geq 1$,

(a) $|\Phi(N)| \prec_{w \log } \Phi(|N|)$ 
(b) $\lambda_{j+k-1}(|\Phi(N)|) \leq \sqrt{\lambda_{j}(\Phi(|N|)) \lambda_{k}(\Phi(|N|))}$

(c) $4 \beta \lambda_{j}\{|\Phi(N)|-\beta \Phi(|N|)\} \leq \lambda_{j}(\Phi(|N|))$.

The weak log-majorisation (a) is well-known, see [3, Theorem 2.9] for a much more general result. (a) and (b) easily follow from the first inequality in the double inequality of the theorem, however (a) and (b) can also be derived from (2.2) and Horn's inequality. (c) is a more subtle estimate, it is a restatement of the first inequality of the theorem. The next corollary extends Proposition 1.1.

Corollary 2.4. If $A$ is a normal matrix, then $\left|A_{\mathbb{R}}\right| \prec_{w \log }|A|_{\mathbb{R}}$.

Proof. Observe that, for any polynomial $p(t)$ with only real coefficients and any $T \in \mathbb{M}_{n}$,

$$
p\left(\bar{T}^{*} \bar{T}\right)=\overline{p\left(T^{*} T\right)}
$$

and so $|\bar{T}|=\overline{|T|}$. Now, consider the normal operator

$$
N=\left(\begin{array}{cc}
A & 0 \\
0 & A
\end{array}\right)
$$

By the previous observation,

$$
|N|=\left(\begin{array}{cc}
|A| & 0 \\
0 & |A|
\end{array}\right)
$$

and applying Corollary 2.3 to $N$ with the positive linear map,

$$
\left(\begin{array}{cc}
X & R \\
L & Y
\end{array}\right) \mapsto \frac{X+Y}{2}
$$

yields $\left|A_{\mathbb{R}}\right| \prec_{w \log }|A|_{\mathbb{R}}$.

Some quite useful positive linear maps are the partial traces on tensor products. Identifying $\mathbb{M}_{d} \otimes \mathbb{M}_{n}$ with $\mathbb{M}_{d}\left(\mathbb{M}_{n}\right)$, the partial trace for the first factor $\operatorname{Tr}_{1}: \mathbb{M}_{d} \otimes \mathbb{M}_{n} \rightarrow$ $\mathbb{M}_{n}$ is defined as

$$
\operatorname{Tr}_{\mathbf{1}}\left(\left(\begin{array}{ccc}
A_{1,1} & \cdots & A_{1, d} \\
\vdots & \ddots & \vdots \\
A_{d, 1} & \cdots & A_{d, d}
\end{array}\right)\right)=\sum_{i=1}^{d} A_{i, i}
$$

Corollary 2.5. Let $N \in \mathbb{M}_{d} \otimes \mathbb{M}_{n}$ be normal. Then, for some unitary $V \in \mathbb{M}_{n}$,

$$
\left|\operatorname{Tr}_{\mathbf{1}}(N)\right| \leq \operatorname{Tr}_{\mathbf{1}}(|N|) \# V \operatorname{Tr}_{\mathbf{1}}(|N|) V^{*}
$$

Applying this corollary to a block-diagonal normal matrix, $N=N_{1} \oplus \cdots \oplus N_{d}$, we obtain the inequality: if $N_{1}, \ldots, N_{d}$ are normal matrices in $\mathbb{M}_{n}$, then for some unitary $V \in \mathbb{M}_{n}$

$$
\left|\sum_{i=1}^{d} N_{i}\right| \leq\left\{\sum_{i=1}^{d}\left|N_{i}\right|\right\} \#\left\{V\left(\sum_{i=1}^{d}\left|N_{i}\right|\right) V^{*}\right\} .
$$


and, consequently,

$$
\left|\sum_{i=1}^{d} N_{i}\right| \leq \frac{1}{2}\left\{\sum_{i=1}^{d}\left|N_{i}\right|+V\left(\sum_{i=1}^{d}\left|N_{i}\right|\right) V^{*}\right\} .
$$

Though the geometric mean inequality is stronger than the arithmetic mean one, the second one may be more natural and useful. For instance, combining with some well-known eigenvalue inequalities for convex functions we may infer from the previous inequality [2, Proposition 2.11].

Let us mention that Theorem 2.2 is optimal: in general, $|\Phi(N)|$ cannot be bounded by a single element into the unitary orbit of $\Phi(|N|)$. In fact, for any $c>0$, there is no general inequality of the form

$$
|\Phi(N)| \leq c V \Phi(|N|) V^{*}
$$

for some unitary $V$. To check that, take $\Phi$ as the partial trace of $\mathbb{M}_{2}\left(\mathbb{M}_{2}\right)$ and set

$$
N=\left(\begin{array}{cc}
P & 0 \\
0 & -Q
\end{array}\right)
$$

where $P, Q \in \mathbb{M}_{2}$ are the projections

$$
P=\left(\begin{array}{ll}
1 & 0 \\
0 & 0
\end{array}\right), \quad Q=\left(\begin{array}{cc}
\cos ^{2} a & \sin a \cos a \\
\sin a \cos a & \sin ^{2} a
\end{array}\right) .
$$

Since

$$
\lim _{a \rightarrow 0^{+}} \frac{\lambda_{2}(\Phi(|N|))}{\lambda_{2}(|\Phi(N)|)}=\lim _{a \rightarrow 0^{+}} \frac{\lambda_{2}(P+Q)}{\lambda_{2}(|P-Q|)}=\lim _{a \rightarrow 0^{+}} \frac{1-\cos a}{\sin a}=0
$$

we infer that (2.4) cannot hold.

\section{$3 \quad$ Some special cases}

The Russo-Dye theorem [6] says that, given a positive linear map $\Phi$ on a unital $C^{*}$ algebra $\mathcal{A}$, we have $\|\Phi(Z)\| \leq\|\Phi(I)\|$ for all contractions $Z \in \mathcal{A}$. We can say much more as stated in the next corollary. We stay in the setting of $\mathbb{M}_{n}$, but the same result holds for an arbitrary unital $C^{*}$-algebra $\mathcal{A}$ acting on a separable Hilbert space $\mathcal{H}$, by considering partial isometries instead of unitaries in $\mathbb{M}_{m}$.

Corollary 3.1. Let $Z \in \mathbb{M}_{n}$ be a contraction and let $\Phi: \mathbb{M}_{n} \rightarrow \mathbb{M}_{m}$ be a positive linear map. Then, for some unitary $V \in \mathbb{M}_{m}$,

$$
|\Phi(Z)| \leq \frac{\Phi(I)+V \Phi(I) V^{*}}{2} .
$$


Proof. We may dilate $Z$ into a unitary $U \in \mathbb{M}_{2}\left(\mathbb{M}_{n}\right)$, for instance with Halmos,

$$
U=\left(\begin{array}{cc}
Z & -\sqrt{I-Z Z^{*}} \\
\sqrt{I-Z^{*} Z} & Z^{*}
\end{array}\right)
$$

Now, let $\Psi: \mathbb{M}_{2}\left(\mathbb{M}_{n}\right) \rightarrow \mathbb{M}_{m}$ be defined as

$$
\Psi\left(\left(\begin{array}{ll}
A & B \\
C & D
\end{array}\right)\right)=\Phi(A)
$$

Applying Theorem 2.2 to $\Psi$ and $U$, we have

$$
|\Phi(Z)|=|\Psi(U)| \leq \frac{\Psi(|U|)+V \Psi(|U|) V^{*}}{2}=\frac{\Phi(I)+V \Phi(I) V^{*}}{2}
$$

for some unitary $V \in \mathbb{M}_{m}$.

Arguing as in the previous proof, and using Corollary 2.3 (a) we have the following log-majorisation:

Corollary 3.2. Let $Z \in \mathbb{M}_{n}$ be a contraction and let $\Phi: \mathbb{M}_{n} \rightarrow \mathbb{M}_{m}$ be a positive linear map. Then

$$
|\Phi(Z)| \prec_{w \log } \Phi(I)
$$

By using a block diagonal matrix $Z=Z_{1} \oplus \cdots \oplus Z_{k}$ and an obvious positive linear map, Corollary 3.2 reads as a folklore result:

Corollary 3.3. Let $X_{1}, \ldots, X_{k}$ be in $\mathbb{M}_{m, n}$ and let $Z_{1}, \ldots, Z_{k}$ be contractions in $\mathbb{M}_{n}$. Then,

$$
\left|\sum_{i=1}^{k} X_{i}^{*} Z_{i} X_{i}\right| \prec_{w \log } \sum_{i=1}^{k} X_{i}^{*} X_{i}
$$

In fact, Corollary 3.3 is rather trivial for an expert since the positivity

$$
\left(\begin{array}{cc}
\sum_{i=1}^{k} X_{i}^{*}\left|Z_{i}^{*}\right| X_{i} & \sum_{i=1}^{k} X_{i}^{*} Z_{i} X_{i} \\
\sum_{i=1}^{k} X_{i}^{*} Z_{i}^{*} X_{i} & \sum_{i=1}^{k} X_{i}^{*}\left|Z_{i}\right| X_{i}
\end{array}\right) \geq 0
$$

entails the factorisation

$$
\sum_{i=1}^{k} X_{i}^{*} Z_{i} X_{i}=\left(\sum_{i=1}^{k} X_{i}^{*}\left|Z_{i}^{*}\right| X_{i}\right)^{1 / 2} K\left(\sum_{i=1}^{k} X_{i}^{*}\left|Z_{i}\right| X_{i}\right)^{1 / 2}
$$

for some contraction $K \in \mathbb{M}_{n}$, so that Corollary 3.3 follows from Horn's inequalities.

A special case of Corollary 3.1 stresses on the role of diagonals for the Schur product with a positive matrix. 
Corollary 3.4. Let $A \in \mathbb{M}_{n}^{+}$and let $Z \in \mathbb{M}_{n}$ be a contraction. If $D$ denotes the diagonal part of $A$, then for some unitary $V \in \mathbb{M}_{n}$,

$$
|A \circ Z| \leq \frac{D+V D V^{*}}{2}
$$

Of course, in Corollary 3.1 and Corollary 3.4 we may use the geometric mean instead of the arithmetic mean.

Another application to Schur product may be recorded.

Corollary 3.5. If $A, B \in \mathbb{M}_{n}$ are normal, then, for some unitary $V \in \mathbb{M}_{n}$,

$$
|A \circ B| \leq|A| \circ|B|+\frac{1}{4} V(|A| \circ|B|) V^{*}
$$

where the constant $1 / 4$ is optimal.

Proof. Note that $A \circ B=\Phi(A \otimes B)$ for some positive linear map (the extraction of a principal submatrix). As $A \otimes B$ is normal and $|A \otimes B|=|A| \otimes|B|$, the inequality follows from Theorem 2.2 with $\beta=1 / 4$. The sharpness of $1 / 4$ is established in (2.3).

The next consequence of our theorem is of a rather general nature, and we state it with the geometric mean.

Corollary 3.6. Let $\Phi: \mathbb{M}_{n} \rightarrow \mathbb{M}_{m}$ be a positive linear map and let $X \in \mathbb{M}_{n}$. Then, for some unitary matrices $U, V \in \mathbb{M}_{m}$,

$$
\left|\Phi\left(X+X^{*}\right)\right| \leq \Phi\left(|X|+\left|X^{*}\right|\right) \# U \Phi\left(|X|+\left|X^{*}\right|\right) U^{*}
$$

and

$$
\left|\Phi\left(X \circ X^{*}\right)\right| \leq \Phi\left(|X| \circ\left|X^{*}\right|\right) \# V \Phi\left(|X| \circ\left|X^{*}\right|\right) V^{*}
$$

Proof. Let $\Psi: \mathbb{M}_{2}\left(\mathbb{M}_{n}\right) \rightarrow \mathbb{M}_{m}$ be defined as

$$
\Psi\left(\left(\begin{array}{ll}
A & B \\
C & D
\end{array}\right)\right)=\Phi(A+B+C+D) .
$$

Since

$$
A+B+C+D=\left(\begin{array}{ll}
I & I
\end{array}\right)\left(\begin{array}{ll}
A & B \\
C & D
\end{array}\right)\left(\begin{array}{l}
I \\
I
\end{array}\right),
$$

$\Psi$ is a positive map. Applying Theorem 2.2 to this map with the normal (Hermitian) operator in $\mathbb{M}_{2}\left(\mathbb{M}_{n}\right)$

$$
\left(\begin{array}{cc}
0 & X \\
X^{*} & 0
\end{array}\right)
$$

yields the first inequality. 
To get the second one, use again that $A \circ B=\Gamma(A \otimes B)$ for some positive linear map $\Gamma$ and pick for $A$ and $B$ the pair of Hermitian operators in $\mathbb{M}_{2 n}$,

$$
A=\left(\begin{array}{cc}
0 & X^{*} \\
X & 0
\end{array}\right), \quad B=\left(\begin{array}{cc}
0 & X \\
X^{*} & 0
\end{array}\right) .
$$

Apply Theorem 2.2 to the normal matrix $A \otimes B$ and the positive linear map $\Phi=\Lambda \circ \Gamma$, where $\Lambda$ is the map on $\mathbb{M}_{2 n}=\mathbb{M}_{2}\left(\mathbb{M}_{n}\right)$ defined as

$$
\Lambda\left(\left(\begin{array}{ll}
S & T \\
Q & R
\end{array}\right)\right)=S+T+Q+R
$$

This yields the second inequality of the Theorem.

Our last illustration of our main result deals with a special class of positive linear maps whose definition will be recalled at the beginning of the proof.

Corollary 3.7. Let $\Phi: \mathbb{M}_{n} \rightarrow \mathbb{M}_{m}$ be a unital two-positive linear map and let $Z \in \mathbb{M}_{n}$ be a contraction. Then,

$$
|\Phi(Z)| \leq \frac{1}{4} I+\Phi(|Z|) .
$$

If $n \geq 3$ and $m \geq 2$, then the constant $1 / 4$ is sharp, even if we confine $\Phi$ to the class of unital completely positive maps and $Z$ to the set of Hermitian contractions.

Proof. $\Phi$ is unital means $\Phi(I)=I$. That $\Phi$ is two-positive means that the linear map on $\mathbb{M}_{2}\left(\mathbb{M}_{n}\right)$,

$$
\left(\begin{array}{ll}
A & B \\
C & D
\end{array}\right) \mapsto\left(\begin{array}{ll}
\Phi(A) & \Phi(B) \\
\Phi(C) & \Phi(D)
\end{array}\right)
$$

is positive. Applying Theorem 2.2 with $\beta=1 / 4$ to this map and a normal (Hermitian) operator in $\mathbb{M}_{2}\left(\mathbb{M}_{n}\right)$ of the form

$$
\left(\begin{array}{cc}
0 & Z \\
Z^{*} & 0
\end{array}\right)
$$

we obtain

$$
\left(\begin{array}{cc}
\left|\Phi\left(Z^{*}\right)\right| & 0 \\
0 & |\Phi(Z)|
\end{array}\right) \leq\left(\begin{array}{cc}
\Phi\left(\left|Z^{*}\right|\right) & 0 \\
0 & \Phi(|Z|)
\end{array}\right)+\frac{1}{4} V\left(\begin{array}{cc}
\Phi\left(\left|Z^{*}\right|\right) & 0 \\
0 & \Phi(|Z|)
\end{array}\right) V^{*}
$$

for some unitary $V \in \mathbb{M}_{2}\left(\mathbb{M}_{n}\right)$. Assuming further that $\Phi$ is (sub)unital we infer the inequality of the theorem,

$$
|\Phi(Z)| \leq \frac{1}{4} I+\Phi(|Z|)
$$

for all contractions $Z \in \mathbb{M}_{n}$.

To check that $1 / 4$ is the best possible in (3.1), consider the map $\Psi: \mathbb{M}_{3} \rightarrow \mathbb{M}_{2}$,

$$
T=\left(\begin{array}{ccc}
t_{1,1} & t_{1,2} & t_{1,3} \\
t_{2,1} & t_{2,2} & t_{2,3} \\
t_{3,1} & t_{3,2} & t_{3,3}
\end{array}\right) \mapsto \Psi(T)=\left(\begin{array}{cc}
t_{1,1} & \frac{1}{2} t_{1,2} \\
\frac{1}{2} t_{2,1} & \frac{1}{4} t_{2,2}+\frac{3}{4} t_{3,3}
\end{array}\right) .
$$


This is a completely positive map as

$$
\left(\begin{array}{cc}
t_{1,1} & t_{1,2} \\
t_{2,1} & t_{2,2}
\end{array}\right) \mapsto\left(\begin{array}{cc}
t_{1,1} & \frac{1}{2} t_{1,2} \\
\frac{1}{2} t_{2,1} & \frac{1}{4} t_{2,2}
\end{array}\right)
$$

is a Schur product with a matrix in $\mathbb{M}_{2}^{+}$. With the Hermitian matrix

$$
H=\left(\begin{array}{lll}
0 & 1 & 0 \\
1 & 0 & 0 \\
0 & 0 & 0
\end{array}\right)
$$

we have

$$
|\Psi(H)|=\frac{1}{4}\left(\begin{array}{ll}
0 & 0 \\
0 & 1
\end{array}\right)+\Psi(|H|)
$$

and so $1 / 4$ cannot be replaced by anything smaller in (3.1).

\section{References}

[1] R. Bhatia, Positive Definite Matrices, Princeton University press, Princeton 2007.

[2] J.-C. Bourin and E.-Y. Lee, Unitary orbits of Hermitian operators with convex or concave functions, Bull. Lond. Math. Soc. 44 (2012), no.6, 1085-1102.

[3] J.-C. Bourin and E.-Y. Lee, Matrix inequalities from a two variables functional, Internat. J. Math. 27, no.9 (2016) 1650071.

[4] M. D. Choi, A Schwarz inequality for positive linear maps, Illinois J. Math., 18, no.4, (1974), 565-574.

[5] C. Davis, A Schwarz inequality for convex operator functions, Proc. Amer. Math. Soc. 8 (1957), 42-44.

[6] B. Russo and H. A. Dye, A note on unitary operators in $C^{*}$-algebras, Duke Math. J. 33 (1966) 413-416.

[7] W. F. Stinespring, Positive functions on $C^{*}$-algebras, Proc. Amer. Math. Soc. 6 (1955), 211-216.

Laboratoire de mathématiques,

Université de Bourgogne Franche-Comté,

25000 Besançon, France.

Email: jcbourin@univ-fcomte.fr

Department of mathematics, KNU-Center for Nonlinear Dynamics,

Kyungpook National University,

Daegu 702-701, Korea.

Email: eylee89@knu.ac.kr 